\title{
Efectos Contextuales, Socioeconómicos y Culturales, sobre los Resultados de México en Lectura en PISA 2009
}

\author{
Contextual, Socioeconomic and Cultural Impacts on the \\ Outcomes of Mexico in Reading in PISA 2009
}

\author{
Eduardo Hernández Padilla * \\ Aldo Bazán Ramírez
}

Universidad Autónoma del Estado de Morelos

\begin{abstract}
La prueba PISA es una evaluación internacional sobre las competencias de lectura, matemáticas y ciencias de los estudiantes de 15 años de edad. En el presente estudio se analizan los efectos que las variables individuales y contextuales tienen en la escala global de lectura y en sus tres dominios durante la aplicación de 2009, en 32 estados de la república mexicana. Mediante el análisis jerárquico multinivel puede apreciarse que las variables contextuales escolares tienen mayor impacto que algunos factores individuales. Estos hallazgos resaltan la importancia que tiene la composición socioeconómica y cultural perteneciente al contexto escolar sobre el desarrollo de las competencias para las sociedades del conocimiento, concretamente la competencia lectora. En contraste, se puede apreciar que los efectos individuales de la mencionada composición son significativamente menores a los contextuales, lo que podría significar la implementación de políticas educativas y sociales, donde se puedan establecer contextos enriquecedores que favorezcan a los alumnos con problemas de aprendizaje en los alumnos de 15 años.
\end{abstract}

Descriptores: Competencia lectora, Factor socioeconómico y cultural, Modelo jerárquico lineal, PISA.

The Programme for International Student Assessment (PISA) is an international assessment of reading literacy, mathematics and science for students 15 years of age. The purpose of this study is to investigate the differences of the effects of socio-economic and cultural status on reading literacy in the global scale and on its three domains during 2009 in 32 states of Mexico. Using hierarchical linear modeling it can be appreciated that the contextual variables have an influence equal to or greater than some individual characteristics. The results highlight the importance of socioeconomic and cultural composition of the school on the development of skills for the knowledge society. In contrast, the individual effects of socioeconomical and cultural composition are significantly lower than contextual, which could mean the implementation of educational and social policies, where they can establish enriching environments that support students with learning disabilities in the students 15 years.

Keywords: Reading literacy, Socio-economic and cultural factor, Hierarchical linear modeling, PISA.

Agradecimiento al Proyecto PROMEP/103.5/13/6626 por el financiamiento otorgado para la realización de este artículo.

*Contacto: eduhpad@gmail.com

Recibido: $\quad 15$ de abril 2015

$1{ }^{\text {a }}$ Evaluación: 1 de junio 2015

ISSN: $1696-4713$

$2^{\text {a }}$ Evaluación: 10 de septiembre 2015

www.rinace.net/reice/

Aceptado: $\quad 30$ de octubre 2015 


\section{Revisión de la Literatura}

La prueba PISA (Programme for International Student Assessment) es una evaluación internacional de habilidades y conocimientos de los estudiantes de 15 años de edad en las áreas de lectura, matemáticas y ciencias; dicha evaluación tiene como objetivo medir hasta qué punto tales alumnos adquirieron conocimientos y habilidades esenciales para la participación efectiva en las sociedades del conocimiento (Organisation for Economic Cooperation and Development-OECD, 2010a). La competencia lectora fue el área de énfasis en las evaluaciones del 2000 y 2009. México ha participado en las mencionadas aplicaciones así como en 2003 y 2012 donde el área principal fue matemáticas, y en 2006 y 2015 que fue ciencias.

$\mathrm{Al}$ igual que en 2000, en PISA 2009 se evaluaron tres aspectos de la competencia lectora: recuperación de la información, interpretación de textos, así como reflexión y evaluación de los mismos. Los resultados de estos tres aspectos fueron combinados en un solo estimado de la competencia lectora global por cada alumno. El análisis de los efectos que tienen diferentes factores, tanto en el puntaje global como en las diferentes subescalas, es el objeto de estudio del presente trabajo.

En acuerdo con tal objetivo, la OECD (2010a) afirma que la educación se encuentra asociada en muchos aspectos a experiencias enriquecedoras en la vida, y particularmente la competencia lectora es uno de los predictores más confiables del bienestar socioeconómico, incluso más que los años de estudio de los padres.

La competencia lectora está conformada por múltiples habilidades cognitivas (la decodificación, el conocimiento de las palabras, la gramática y las estructuras y las características textuales y lingüísticas, además del conocimiento del mundo), así como algunas de tipo metacognoscitivo (como el uso de estrategias apropiadas al leer los textos). De acuerdo con PISA en 2009,"la competencia lectora (es) la capacidad de un individuo para comprender, emplear, reflexionar e interesarse en textos escritos con el fin de lograr metas propias, desarrollar sus conocimientos y su potencial personal, y participar en la sociedad” (Díaz y Flores, 2010, p. 40).

La escala completa de lectura se conformó de tres componentes: Acceder y Recuperar, Integrar e Interpretar y Reflexionar y Evaluar. El dominio Acceder y recuperar se refiere al ingreso al texto en donde se encuentra la información y su correspondiente obtención; por su parte, Integrar e interpretar (lo que se lee) refiere a comprender las relaciones internas del texto y darles sentido. Finalmente, el dominio Reflexionar y evaluar es entendido como tomar el texto a modo de base y relacionarlo con la experiencia propia para emitir un juicio.

Los resultados en lectura a nivel nacional de México en la prueba PISA entre 2000 y 2009 muestran que hay una disminución de cuatro puntos porcentuales de los estudiantes de 15 años que obtuvieron una calificación en los niveles bajos de la prueba (por debajo del nivel dos, de un rango de 407.7 puntos y por debajo de éstos). La subescala de Reflexionar y Evaluar muestra un incremento marginal de un punto porcentual en los niveles bajos, en tanto que en Integrar e Interpretar, así como en Acceder y Recuperar hay un descenso del número de estudiantes que están en dichos niveles bajos. En particular, en esta última subescala, Acceder y Recuperar, sobresalen 
los resultados debido a que hay una disminución de un $14 \%$ de alumnos con un nivel insatisfactorio, mientras que hay un incremento de $7 \%$ en los niveles 3 y 4 y mayores (véase la figura 6.6 de Díaz y Flores, 2010, para los datos completos).

La mejora en los puntajes de la prueba PISA coincide con la puesta en marcha del Programa Nacional de Lectura y Escritura (PNLE) por la Secretaria de Educación Pública en el año 2002, así como la Reforma en Educación Secundaria en el 2006. Dicho programa tiene entre sus objetivos la movilización social en favor de la cultura escrita en la escuela y fuera de ella, para la participación de la comunidad escolar y de la sociedad, en donde una línea estratégica ha sido el fortalecimiento de los acervos bibliográficos de las escuelas así como la capacitación de los diversos actores en el escenario escolar. Sin embargo, la información sobre el aprovechamiento escolar muestra que, pese a los avances logrados en los años recientes, los niveles de logro alcanzados en la educación básica se encuentran por debajo de lo esperado, por lo que es necesario reconocer la adquisición insuficiente de competencias básicas, la lectura entre ellas (Cámara de Diputados, 2013).

De igual manera, pese a los esfuerzos realizados por los gobiernos federal y estatales, diversos estudios sobre el aprendizaje de alumnos en áreas afines a la lectura, como es el español, demuestran amplias brechas educativas entre la población que recibe educación básica; estas brechas son atribuibles principalmente al ambiente socioeconómico y cultural de la familia del estudiante (Backhoff, Andrade, Sánchez, Peon y Bouzas, 2006; Backhoff, Bouzas, Contreras, Hernández y García, 2007; Backhoff, Bouzas, Hernández y García, 2007). Los resultados de logro educativo evidencian bajos indicadores de calidad, pese a la constante mejora de planes y programas de estudio, $\mathrm{y}$ a las diversas reformas a la educación básica en México (Bazán, Barrera y Vega, 2013; Vázquez, 2005). A continuación se revisan algunos de los factores influyentes en el logro educativo.

\section{Influencia de factores individuales y contextuales en el desempeño académico}

El desempeño o aprendizaje escolar están sujetos a la influencia de diversas variables que inciden positiva o negativamente sobre aquél; dichas variables pueden ser relativas al individuo, como las provenientes del contexto familiar, o bien escolares, como el ambiente escolar o la suma de las aportaciones del nivel socioeconómico de cada uno de los alumnos al de la escuela. Dentro de este ámbito de factores influyentes, el informe de Coleman y colegas (1966) fue trascendental debido a que concluía que ni la escuela o sus recursos, así como ni el currículo, tienen una influencia significativa sobre el desempeño académico de los alumnos, por el contrario, el nivel social, económico y cultural de las familias y del contexto son las fuentes de desigualdades en el rendimiento (Lizasoain, Jorasti, Lukas y Santiago, 2007). Como consecuencia directa de esta aseveración la escuela pasó a ser vista como una reproducción del sistema de privilegios sociales.

Por su parte, diversos estudios han evidenciado que las variables individuales influyen de manera significativa en el desempeño de las diferentes competencias que evalúa la prueba PISA. Los efectos que tienen el entorno socioeconómico del alumno muestran que hay efectos diferenciales sobre su desempeño educativo. En el caso de lectura, el nivel educativo de los padres (pero no el de la madre), el estatus ocupacional de los padres y la disponibilidad de recursos educativos en el lugar (diccionario, texto de estudio, etc.) tienen un efecto favorable en el rendimiento escolar; en tanto que en el caso de matemáticas y ciencias, los factores que influyen decisivamente en el desempeño es el estatus ocupacional de los padres (ambos); la riqueza familiar, bienes y servicios; y el 
nivel educativo de los padres (Carrasco, 2008). Sin embargo, es de señalar que la relación entre nivel socioeconómico y el desempeño académico depende de la definición del primero (Sirin, 2005).

A este último respecto, el nivel socioeconómico ha sido expresado como el capital que posee una familia, el cual influye indirectamente en el rendimiento escolar de los estudiantes. De hecho, el término denomina las ventajas culturales y sociales que los individuos o las familias poseen $\mathrm{y}$ por consiguiente los conducen a un nivel socioeconómico más elevado, aunque las limitaciones en el concepto de capital económico para explicar la relación entre éste y el desempeño escolar, es lo que lleva a considerar otras formas de capital como el social y el cultural que interactúan con el capital económico, y que influyen en el rendimiento (Backhoff et al., 2007; Bonamino y Creso, 2004). Backhoff y colaboradores (2007) señalan que sólo a través de un buen capital económico es posible acceder a un capital cultural objetivado, el cual existe en la forma de bienes culturales, acceso a servicios específicos.

Es plausible esperar que familias que tienen un capital económico elevado proporcionen a sus hijos acceso a excelentes instituciones educativas, a viajes de estudio, la garantía de un lugar apropiado para estudiar entre otras cosas (Coleman et al., 1966); en tanto que para el capital social los elementos que los constituyen son las redes de relaciones sociales (las cuales permiten a un individuo tener acceso a los recursos del grupo social), y la cantidad y la calidad de recursos del grupo (social) (OECD, 2010b). Por su parte, otros autores indican que tanto el capital cultural como el nivel socioeconómico y educativo de los padres no propician un mejor aprovechamiento educativo de lo que lo hacen variables de tipo actitudinal y de competencia como el interés de los padres por la educación de sus hijos y las expectativas que se generan sobre esta última (Bazán, Sánchez y Castañeda, 2007; Cervini, 2002).

La visión de la escuela como un sistema de producción compuesto por cuatro elementos correlacionados entre sí (el contexto, las entradas, el proceso educativo y los resultados) (López, Navarro, Ordóñez y Romero, 2009), ubica a la escuela como unidad productora de resultados educativos requiere de una serie de recursos escolares necesarios para su consecución, como son las instalaciones, el equipamiento, el personal, etc., que deben ser eficientemente empleados para alcanzar esos logros educativos que se verán reflejados, entre otras cosas, en las competencias necesarias para la vida (López et al., 2009). De esto puede deducirse que el contexto es uno de los factores que ejercen una gran influencia en el rendimiento académico, y por ello se puede considerar como un elemento clave de la calidad educativa.

Sin embargo, no todas las variables del centro escolar influyen significativamente sobre el aprendizaje de los estudiantes. Hanushek (1989) analizó la relación entre los gastos básicos en educación y el rendimiento de los alumnos sin encontrar que factores como el número de alumnos por profesor, la formación del profesorado o su experiencia tengan algún efecto sobre el desempeño de los alumnos .En tanto que Backhoff y otros (2006), tampoco encontraron efectos significativos de la cobertura curricular, del clima escolar, de la infraestructura en el logro educativo de los estudiantes de tercero de secundaria.

Cabe señalar que los resultados de estos trabajos están realizados en estudios a gran escala; los análisis de trabajos que emplean muestras pequeñas y que analizan la eficacia escolar (entendida ésta como la que consigue la escuela en el desarrollo integral de todos los estudiantes independientemente de la situación socioeconómico y cultural de sus 
familias), muestran un alto grado de coherencia en los factores escolares asociados al desarrollo de los estudiantes, los cuales son positivos o negativos y afectan la eficacia escolar. Estos factores generalmente no son analizados en las evaluaciones a gran escala y son: clima escolar y del aula; sentido de comunidad; liderazgo educativo; altas expectativas; calidad del currículo y las estrategias de enseñanza; organización del aula; seguimiento y evaluación; desarrollo profesional de los docentes; implicación de las familias; e, instalaciones y recursos (Hernández-Castilla, Murillo y Martínez-Garrido, 2014).

La literatura acerca de la influencia del contexto escolar sobre el desempeño en las diferentes competencias de PISA, sin ser abundante, sí evidencia efectos significativos; tal es el caso de matemáticas en donde el contexto puede explicar hasta un dígito el porcentaje de variación entre estudiantes, y casi un 90\% entre escuelas (Langer, 2007). Cabe mencionar que estos son resultados de Luxemburgo donde la elección del tipo de escuela secundaria es controlada y las variables contextuales, en consecuencia, no varían dentro de las escuelas sino entre ellas.

Por otro lado, existe investigación que apunta a que, entre todas las variables relativas a los centros escolares (tamaño de la escuela o aula, grado de autonomía de las escuelas, clima escolar, el nivel y uso de los recursos y la tecnología, entre otros), es el nivel socioeconómico promedio del centro educativo el que más influencia tiene sobre el rendimiento académico de los alumnos (Carrasco, 2008).

En los estudios sobre PISA los resultados muestran que hay una relación positiva entre el gasto en instituciones educativas por estudiante y el producto doméstico bruto per cápita tanto en los niveles primario y secundario de educación; los países más pobres de la OECD tienden a gastar menos por estudiante que los más ricos. Otro de los indicadores importantes de los recursos destinados a la educación es la razón de estudiantes a personal de enseñanza, donde México tiene una de las mayores razones de los países de la OECD; en tanto que en el salario destinado a profesores con más de 15 años de experiencia, nuestro país ocupa uno de los últimos lugares, pese a que el salario y las condiciones de trabajo son importantes para atraer, desarrollar y retener maestros habilidosos y efectivos. De igual forma, la centralización de las decisiones está vinculada con los países menos exitosos, en tanto que los que obtienen mejores promedios nacionales, sus autoridades están descentralizadas (Aydin, Uysal y Sarier, 2010).

Asimismo, los resultados de PISA muestran que el nivel socioeconómico medio de una escuela tiene un efecto sobre el rendimiento del estudiante, efecto que se superpone y supera a las influencias asociadas al propio nivel socioeconómico familiar del estudiante (Lizasoain et al., 2007). La posesión de un capital cultural común en la escuela (efecto contextual) favorece el desempeño escolar en la medida que facilita el aprendizaje de los contenidos legítimos (cultos apropiados), que son llevados del hogar a la escuela por los niños, en la medida que funcionan entre el mundo familiar y la cultura escolar; todo esto conduce a que la educación escolar, de niños culturalmente favorecidos, es una continuación de la educación familiar.

En el caso de México, las pruebas estandarizadas como el Examen para la Calidad y el Logro Educativo (Excale) han mostrado reiteradamente que los alumnos que asisten a las escuelas privadas tienen un desempeño académico por encima de sus pares que acuden a instituciones públicas (Sánchez y Andrade, 2009, 2013), efectos atribuibles al 
contexto escolar incluso cuando se ha controlado la influencia del estatus sociocultural de la familia (Backhoff et al., 2006, 2007).

El objetivo del análisis en el presente estudio fue relacionar el logro en la competencia lectora con un conjunto de variables que se consideran relevantes, esto con la finalidad de conocer cuáles de ellas son efectivas en ambos niveles, el del estudiante y el de la escuela, tanto en la escala global de lectura como en las tres diferentes escalas que se conforman la escala global.

El procedimiento seguido fue proponer diversos modelos jerárquicos lineales en los que se introducen diversas variables que tienen como finalidad el responder a hipótesis específicas. El modelo nulo o vacío no tiene alguna variable a nivel del estudiante o al de la escuela que predigan el desempeño en lectura, y las subescalas que conforman la escala global. El modelo sirve como referencia para conocer el impacto de las variables que son paulatinamente ingresadas en los modelos subsecuentes, los cuales responden a preguntas específicas. El modelo siguiente, incluye el índice sobre nivel socioeconómico y cultural (ESCS por sus siglas en inglés) del estudiante; este modelo responde a la interrogante sobre los diferenciales individuales atribuibles a este índice. El tercer modelo, además del índice ESCS, incluye la repetición en la educación primaria y en la secundaria, el sexo (teniendo como valor de referencia los hombres), el nivel educativo de los estudiantes (con la secundaria como valor de referencia), asimismo, incluye el tiempo y el gusto por la lectura al nivel del alumno; el modelo tiene como finalidad conocer los efectos que algunas de las variables individuales del alumno ejercen sobre el desempeño en la competencia lectora. El cuarto y último modelo, además de las variables ya enunciadas, incorpora algunas variables del nivel de escuela como son el tipo de sostenimiento, el promedio del nivel socioeconómico y cultural de la escuela (ESCS), así como su desviación estándar; estas últimas a fin de conocer cómo influyen los efectos contextuales de la escuela y su heterogeneidad impactan sobre el desempeño del estudiante en la competencia global de lectura y los diferentes dominios que la conforman.

Ninguna otra variable al nivel del alumno o de la escuela fue usada, por su parte todas las pendientes fueron modeladas de forma fija; y ningún término de interacción fue analizado. La significatividad de los coeficientes de regresión individual es empleada para decidir si la contribución de cada uno de ellos al modelo es relevante o aporta algo al mismo.

Acorde con lo expuesto anteriormente, el análisis mediante los modelos jerárquicos lineales sobre el rendimiento en la prueba de lectura se guio por las siguientes hipótesis.

- Existe una variación significativa del rendimiento promedio entre los alumnos asociada al nivel socioeconómico y cultural de la familia.

- La capacidad de explicación de las variables individuales y familiares de los individuos son mayores que al poder explicativo del factor socioeconómico y cultural, exclusivamente.

- El contexto económico, cultural y social se asocia significativamente con su rendimiento, además de que su capacidad explicativa es superior a los de la familia. 


\section{Método}

Para la realización de esta investigación se utilizaron los datos de 38250 alumnos mexicanos provenientes de 1535 escuelas que participaron en la prueba de lectura de PISA en la aplicación de 2009, los cuales constituyen la muestra representativa a nivel nacional y estatal.

\subsection{Variables}

El cuestionario del estudio PISA además de evaluar el desempeño de los estudiantes en las prueba de lectura, matemáticas y ciencias, también recolecta información de aspectos socioeconómicos y culturales del alumno y de la familia, así como de otros factores que ayudan a explicar las diferencias en los resultados educativos.

La principal variable de interés fue el nivel socioeconómico y cultural del niño (ESCS) debido a que ésta es una variable que ha demostrado ser siempre significativa en los resultados; otras variables al nivel del alumno y de la escuela fueron después introducidas en el modelo. El índice ESCS estuvo conformado por el más alto nivel laboral de los padres, por el más alto nivel de estudio de los padres, expresado en años de escolaridad, y la escala de posesiones en el hogar. El índice tiene una media de cero para el estudiante, el cual es el promedio de los países que integran la OECD, y una desviación estándar igual a uno $(\mathrm{OECD}, 2012)$. En el caso de México la media del ESCS es de -1.12 y una desviación estándar de 1.2.

Se han encontrado efectos de influencia significativa de ESCS en la competencia lectora, además de la habilidad para leer textos digitales (Lee y Wu, 2012), y en el desempeño en matemáticas (Langer, 2007).

La variable sexo fue introducida en los diferentes modelos al nivel del alumno donde el valor de referencia son los hombres, esto se debe a que en diversos estudios las mujeres obtienen en promedio mejores puntajes que aquellos en la escala global de lectura y sus diferentes dominios (Díaz y Flores, 2010; OECD, 2010a), aunque la diferencia entre los países que conforman la OCDE no son consistentes ni constantes (Yip, Chiu y Ho, 2004). Asimismo, se introducen las variables de reprobación en la primaria y en la secundaria, las cuales han mostrado tener una importancia significativa en el logro educativo (Backhoff et al., 2006). También se incluyen las horas de estudio dedicadas a la lectura, y un índice de gusto por la misma.

El indicador del tipo de sostenimiento de la escuela tuvo como valor de referencia el sostenimiento público. Cabe señalar que los costos de las escuelas privadas son casi enteramente cubiertos por los padres o por donaciones; consecuentemente el ingreso a las escuelas privadas es en gran medida determinadas por la clase social; aunque en México ante la oferta insuficiente del servicio de educación media superior hay un número significativo de escuelas que imparten este nivel educativo y cuya calidad es muy variable (Robles et al., 2013).

Las variables contextuales (a nivel de la escuela) se formaron mediante el promedio de cada variable individual del alumno y la desviación estándar de la misma, esta última representa la heterogeneidad en la composición de la escuela, a saber, a mayor valor de la desviación estándar del ESCS mayor variabilidad en su conformación.

A partir del conjunto de variables citadas anteriormente se utilizan diversos modelos en los que se empleó como variable dependiente el rendimiento en lectura de los alumnos de 
15 años, a través de sus cinco valores plausibles, tanto en la escala global como en sus tres subcomponentes.

\subsection{Técnica de análisis}

Los estudiantes dentro de las escuelas conforman una estructura jerárquica de datos, por lo que las observaciones no son independientes unas de otras, lo cual es asumido por la teoría estadística estándar. Por lo anterior los modelos clásicos de regresión producen sesgos en la estimación de las características del estudiante y de la escuela.

El modelo jerárquico lineal (Raudenbush y Bryk, 2002) ofrece a la investigación en el ámbito educativo la posibilidad de poder recoger la estructura anidada de los datos educativos en sus niveles de alumnos y de escuelas, así como poder distinguir con mayor precisión los efectos debidos a cada uno de esos niveles.

El ordenamiento secuencial del estudio responde a la técnica de análisis y a la hipótesis propuesta; se comienza del modelo nulo hasta llegar a uno más complejo, es decir, la agregación de variables se realiza de manera gradual. Se inicia con la partición de la varianza del rendimiento por niveles (primer paso), luego se continua modelando la asociación entre el rendimiento por una parte, y el índice de nivel socioeconómico y cultural (segundo paso), para dar paso a los indicadores individuales del alumno (tercer paso), más los del contexto escolar (cuarto paso).

- Primer paso: Estimación del modelo nulo. Este es el primer paso que permitió determinar la proporción de las desigualdades en el rendimiento y que se explica principalmente por factores que operan a través del agrupamiento escolar (diferencias entre escuelas) y a nivel individual (distinciones entre alumnos).

- Segundo paso: Modelo nivel socioeconómico y cultural individual. En este segundo paso se tuvo como objetivo conocer la influencia que el nivel socioeconómico y cultural individual del estudiante tiene sobre su logro en lectura.

- Tercer paso: Modelo de variables individuales. En este paso tuvo como finalidad determinar el impacto de las características individuales del alumno (repetición, sexo, nivel educativo, horas dedicadas a la lectura y un índice de gusto por la lectura) sobre su logro escolar, una vez controlado el índice de nivel socioeconómico y cultural del estudiante.

- Cuarto paso: Modelos de efectos contextuales. En este último paso se evaluó el grado de asociación entre cada medición individual y "contextual” con el rendimiento en lectura, considerando a todas ellas simultáneamente.

Debe mencionarse que todas las variables métricas fueron centradas respecto a la gran media a fin de evaluar posteriormente contra la escuela promedio del país, y que, como se señaló previamente, el grupo de referencia consistió de estudiantes mexicanos hombres de 15 años que asisten a escuelas secundarias públicas, con el promedio nacional de ESCS, sin reporte de repetición en primaria y/o secundaria. 


\section{Resultados}

En el presente estudio se analizaron los resultados de la prueba PISA 2009 en la competencia lectora en México en cuatro diferentes modelos jerárquicos lineales. El objetivo de dichos análisis fue conocer el aporte que hacen tanto las características individuales de los alumnos así como de las condiciones contextuales de la escuela en el desempeño de los estudiantes en la escala global de lectura, y en las tres subescalas que la conforman. Finalmente el análisis de las varianzas entre escuelas indica las diferencias entre las mismas toda vez que se han ingresado variables explicativas a los distintos modelos.

En la tabla 1, se muestra que el índice ESCS del alumno en el modelo sobre escala global es muy reducido, menos de 10 puntos por cada desviación estándar del índice; esta contribución se mantiene relativamente estable a lo largo de los diferentes modelos. En el modelo de variables individuales se observa que repetir un grado escolar en primaria afecta negativamente el resultado en la escala global en casi 50 puntos, en tanto que la repetición en secundaria sólo disminuye 20 puntos. El ubicarse en educación media superior mejora el puntaje del alumno 20 puntos en comparación de sus contrapartes que se ubican en la enseñanza secundaria.

Tabla 1. Modelos pertenecientes a la escala global de lectura en la prueba PISA 2009

\begin{tabular}{|c|c|c|c|c|c|c|c|c|}
\hline & \multicolumn{2}{|c|}{ M. nulo } & \multicolumn{2}{|c|}{$\begin{array}{c}\text { M. nivel } \\
\text { socioeconómico y } \\
\text { cultural individual }\end{array}$} & \multicolumn{2}{|c|}{$\begin{array}{c}\text { M. de } \\
\text { variables } \\
\text { individuales }\end{array}$} & \multicolumn{2}{|c|}{$\begin{array}{l}\text { M. de efectos } \\
\text { contextuales }\end{array}$} \\
\hline & Est. & EE & Est. & $\mathbf{E E}$ & Est. & $\mathbf{E E}$ & Est. & EE \\
\hline Intercepto & $389,5^{*}$ & 3,7 & $398,5^{*}$ & 4,2 & $415,2^{*}$ & 4,4 & $423,3^{*}$ & 3,7 \\
\hline ESCS & & & $5,2^{*}$ & 0,9 & $8,3^{*}$ & 0,7 & $6,5^{*}$ & 0,7 \\
\hline Repetición primaria & & & & & $-47,9^{*}$ & 3,5 & $-46,6^{*}$ & 3,5 \\
\hline Repetición secundaria & & & & & $-13,2$ & 6,8 & $-15,1^{*}$ & 6,6 \\
\hline Sexo & & & & & $9,0^{*}$ & 1,6 & $8,7 *$ & 1,6 \\
\hline Nivel educativo & & & & & $35,1^{*}$ & 4,7 & $23,4^{*}$ & 4,1 \\
\hline Horas de lectura & & & & & $-1,2$ & 1,5 & $-1,0$ & 1,5 \\
\hline Gusto por la lectura & & & & & $8,7 *$ & 0,6 & $8,8^{*}$ & 0,6 \\
\hline $\begin{array}{l}\text { Promedio de ESCS de la } \\
\text { escuela }\end{array}$ & & & & & & & $30,3^{*}$ & 2,5 \\
\hline $\begin{array}{l}\text { Desviación estándar de } \\
\text { ESCS de la escuela }\end{array}$ & & & & & & & $-32,9^{*}$ & 10,4 \\
\hline Sostenimiento & & & & & & & $-12,2^{*}$ & 5,3 \\
\hline $\begin{array}{l}\text { Porcentaje de la varianza } \\
\text { en los alumnos }\end{array}$ & 36, & & & & 69 & & 80 & \\
\hline $\begin{array}{l}\text { Porcentaje de la varianza } \\
\text { entre escuelas }\end{array}$ & 63 , & & & & 30 , & & 20 & \\
\hline
\end{tabular}

Nota: ${ }^{*} \mathrm{p}<0,05$.

Fuente: Elaboración propia con datos de la aplicación de PISA (2009).

Por su parte, el modelo referido como contextual muestra que hay un incremento en más de 10 puntos en el intercepto, en tanto que el efecto de ESCS crece casi cinco puntos. Un cambio notable ocurre en las variables de repetición: mientras que reprobar un grado en primaria afecta 48 puntos la calificación global, la reprobación en secundaria deja de ser significativa. En tanto que el cursar el primer año de la educación media superior mejora en 35 puntos con respecto a secundaria, el ser mujer sólo lo hace en nueve puntos; una mejora equivalente que proporciona el índice del gusto por la lectura (8.7 puntos), aunque las horas dedicadas a la misma no influyen de manera significativa. El modelo de 
efectos contextuales muestra que, una vez controlados los efectos del nivel socioeconómico y cultural individual así como de las variables individuales (donde estas últimas muestran pequeñas variaciones de éstas respecto al anterior modelo, con dos importantes excepciones: la repetición en secundaria se vuelve significativa, menos 15 puntos, y el nivel educativo disminuye 12 puntos), el ambiente promedio de nivel socioeconómico y cultural de la escuela es favorecedor, siendo la mejora de 30 puntos, en tanto que la composición heterogénea de las escuelas afecta negativamente (33 puntos) el rendimiento en la escala global de lectura. Finalmente, la asistencia a las escuelas privadas afecta en 12 puntos el desempeño en la competencia global.

En la subescala de Acceder y Recuperar la media general es ligeramente mayor que en la escala global por seis puntos. En tanto que en el modelo sobre el nivel socioeconómico y cultural el valor del intercepto disminuye marginalmente un punto, mientras que el índice ESCS aporta cinco puntos al resultado general. En el modelo de variables individuales, cuando se controlan las mismas, el valor de la media incrementa 15 puntos respecto al modelo previo, en tanto que el índice ESCS lo hace en tres puntos; por su parte, los aportes que hacen las mencionadas variables individuales muestra que para esta escala el efecto de la reprobación escolar es significativa en primaria y secundaria (menos 53 y 17 puntos, respectivamente), mientras que el ser mujer tiene una contribución pequeña (cinco puntos), algo semejante ocurre con el gusto por la lectura (nueve), solamente el nivel educativo de media superior hace una aportación relevante con casi 40 puntos; finalmente, las horas de lectura no fueron estadísticamente significativas (tabla 2 ).

Tabla 2. Modelos pertenecientes a la subescala Acceder y recuperar en la prueba PISA 2009

\begin{tabular}{|c|c|c|c|c|c|c|c|c|}
\hline & \multicolumn{2}{|c|}{ M. nulo } & \multicolumn{2}{|c|}{$\begin{array}{c}\text { M. nivel } \\
\text { socioeconómico y } \\
\text { cultural individual }\end{array}$} & \multicolumn{2}{|c|}{$\begin{array}{c}\text { M. de } \\
\text { variables } \\
\text { individuales }\end{array}$} & \multicolumn{2}{|c|}{$\begin{array}{l}\text { M. de efectos } \\
\text { contextuales }\end{array}$} \\
\hline & Est. & EE & Est. & $\mathbf{E E}$ & Est. & $\mathbf{E E}$ & Est. & EE \\
\hline Intercepto & $395,7 *$ & 4,1 & $398,7^{*}$ & 4,2 & $414,3^{*}$ & 4,9 & $424,4^{*}$ & 4,3 \\
\hline ESCS & & & $4,7^{*}$ & 1,2 & $7,9^{*}$ & 0,8 & $5,7^{*}$ & 0,9 \\
\hline Repetición primaria & & & & & $-52,9^{*}$ & 5,0 & $-51,5^{*}$ & 5,0 \\
\hline Repetición secundaria & & & & & $-16,8^{*}$ & 8,2 & $-18,7^{*}$ & 8,1 \\
\hline Sexo & & & & & $4,9^{*}$ & 2,1 & $4,6^{*}$ & 2,1 \\
\hline Nivel educativo & & & & & $38,6^{*}$ & 5,2 & $27,7^{*}$ & 1,6 \\
\hline Horas de lectura & & & & & $-1,5$ & 1,7 & $-1,3$ & 1,7 \\
\hline Gusto por la lectura & & & & & $9,0^{*}$ & 0,7 & $9,2^{*}$ & 0,7 \\
\hline $\begin{array}{l}\text { Promedio de ESCS de la } \\
\text { escuela }\end{array}$ & & & & & & & $31,1^{*}$ & 2,5 \\
\hline $\begin{array}{l}\text { Desviación estándar de } \\
\text { ESCS de la escuela }\end{array}$ & & & & & & & $-40,1^{*}$ & 11,8 \\
\hline Sostenimiento & & & & & & & $-16,0^{*}$ & 5,9 \\
\hline $\begin{array}{l}\text { Porcentaje de la varianza } \\
\text { en los alumnos }\end{array}$ & & 41,1 & & 42,8 & & 74,2 & & 82,5 \\
\hline $\begin{array}{l}\text { Porcentaje de la varianza } \\
\text { entre escuelas }\end{array}$ & & 58,9 & & 57,2 & & 25,8 & & 17,5 \\
\hline
\end{tabular}

Nota: $* \mathrm{p}<0,05$.

Fuente: Elaboración propia con datos de la aplicación de PISA (2009).

Los modelos generados para la subescala de Integrar e Interpretar son presentados en la tabla 3. El valor de los interceptos en los diferentes modelos son menores a los obtenidos en la escala global y la subescala Acceder y Recuperar, aunque hay un incremento 
paulatino conforme se introducen variables en los mismos. El valor de ESCS es aproximadamente de cinco puntos en el modelo de nivel socioeconómico y cultural individual, aunque en los modelos subsecuentes es mayor. La repetición en primaria afecta de manera negativa y significativa el desempeño en la subescala en los modelos de variables individuales (46 puntos), y el contextual (45 puntos); en cuanto a la reprobación en secundaria no es estadísticamente significativa en el modelo variables individuales, pero sí en el contextual, en el que se afecta negativamente con 14 puntos.

El ser mujer mejora en 10 puntos los resultados de la subescala en el modelo de variables individuales, en tanto que asistir a la educación media superior tiene una influencia significativa y positiva de 35 , aunque este efecto disminuye en el modelo de efectos contextuales a 23 puntos. En lo que a actividades de lectura se refiere no hay una influencia significativa en el número de horas dedicadas, aunque sí en la buena disposición a la lectura: nueve puntos en los modelos de variables individuales y contextuales. Finalmente, los efectos contextuales son significativos tanto en el promedio de ESCS de la escuela (31 puntos), como en la heterogeneidad de la composición de la escuela (menos 32 puntos), y en el tipo de sostenimiento privado (menos 11).

Tabla 3. Modelos pertenecientes a la subescala Integrar e interpretar en la prueba PISA 2009

\begin{tabular}{|c|c|c|c|c|c|c|c|c|}
\hline & \multicolumn{2}{|c|}{ M. nulo } & \multicolumn{2}{|c|}{$\begin{array}{c}\text { M. nivel } \\
\text { socioeconómico y } \\
\text { cultural individual }\end{array}$} & \multicolumn{2}{|c|}{$\begin{array}{c}\text { M. de } \\
\text { variables } \\
\text { individuales }\end{array}$} & \multicolumn{2}{|c|}{$\begin{array}{l}\text { M. de efectos } \\
\text { contextuales }\end{array}$} \\
\hline & Est. & EE & Est. & $\mathbf{E E}$ & Est. & EE & Est. & EE \\
\hline Intercepto & $3821 *$ & 37 & $3852^{*}$ & 37 & $3971 *$ & 40 & $4078^{*}$ & 34 \\
\hline ESCS & & & $53^{*}$ & 11 & $89^{*}$ & 08 & $69^{*}$ & 08 \\
\hline Repetición primaria & & & & & $-460^{*}$ & 38 & $-445^{*}$ & 39 \\
\hline Repetición secundaria & & & & & -123 & 70 & $-143^{*}$ & 69 \\
\hline Sexo & & & & & $95^{*}$ & 16 & $92 *$ & 16 \\
\hline Nivel educativo & & & & & $350^{*}$ & 47 & $227 *$ & 41 \\
\hline Horas de lectura & & & & & -11 & 14 & -09 & 14 \\
\hline Gusto por la lectura & & & & & $90^{*}$ & 07 & $92^{*}$ & 07 \\
\hline $\begin{array}{l}\text { Promedio de ESCS de la } \\
\text { escuela }\end{array}$ & & & & & & & $314 *$ & 26 \\
\hline $\begin{array}{l}\text { Desviación estándar de } \\
\text { ESCS de la escuela }\end{array}$ & & & & & & & $-323^{*}$ & 99 \\
\hline Sostenimiento & & & & & & & $-118^{*}$ & 60 \\
\hline $\begin{array}{l}\text { Porcentaje de la varianza } \\
\text { en los alumnos }\end{array}$ & & 381 & & 405 & & 701 & & 804 \\
\hline $\begin{array}{l}\text { Porcentaje de la varianza } \\
\text { entre escuelas }\end{array}$ & & 619 & & 595 & & 299 & & 196 \\
\hline
\end{tabular}

Nota: * $\mathrm{p}<005$.

Fuente: Elaboración propia con datos de la aplicación de PISA (2009).

En la subescala Reflexionar y Evaluar los interceptos de los diferentes modelos son mayores que en la subescala Integrar e interpretar, además de que muestran un crecimiento paulatino en aquéllos. Por su parte, el índice ESCS a nivel individual tiene un comportamiento análogo al de las subescalas previas: en el modelo socioeconómico y cultural individual el valor es de cinco unidades, el cual crece dos puntos en el modelo de variables individuales, para volver a disminuir a cinco puntos en el modelo contextual. Para el modelo de variables individuales, la repetición en primaria afecta de manera significativa y negativa en 49 puntos aproximadamente el desempeño, mientras que la 
reprobación en secundaria no es significativa. En tanto que el ser mujer mejora 11 puntos el desempeño en la subescala, y la estadía en la educación media superior mejora en 32 puntos el logro educativo. Finalmente, en el modelo de efectos contextuales, la reprobación en el nivel de primaria incide negativamente en el logro (menos 47 puntos), en tanto que el sexo y el nivel educativo disminuyen el efecto con respecto al modelo previo, aunque el primero lo hace de manera marginal (10.7 y 22, en ese orden). Nuevamente, el promedio del ESCS de la escuela hace un aporte significativo al logro (28 puntos); por su parte la heterogeneidad de la composición de la misma afecta negativamente el desempeño en esta subescala (menos 32 puntos). El tipo de sostenimiento no influye (ver tabla 4).

Tabla 4. Modelos pertenecientes a la subescala Integrar e interpretar en la prueba PISA 2009

\begin{tabular}{|c|c|c|c|c|c|c|c|c|}
\hline & \multicolumn{2}{|c|}{ M. nulo } & \multicolumn{2}{|c|}{$\begin{array}{l}\text { M. nivel } \\
\text { socioeconómico y } \\
\text { cultural individual }\end{array}$} & \multicolumn{2}{|c|}{$\begin{array}{c}\text { M. de } \\
\text { variables } \\
\text { individuales }\end{array}$} & \multicolumn{2}{|c|}{$\begin{array}{l}\text { M. de efectos } \\
\text { contextuales }\end{array}$} \\
\hline & Est. & EE & Est. & EE & Est. & EE & Est. & EE \\
\hline Intercepto & $395,5^{*}$ & 3,9 & $398,7^{*}$ & 4,0 & $415,2^{*}$ & 4,5 & $424,1 *$ & 4,2 \\
\hline ESCS & & & $5,8^{*}$ & 1,1 & $8,5^{*}$ & 0,8 & $6,7^{*}$ & 0,8 \\
\hline Repetición primaria & & & & & $-48,5^{*}$ & 3,9 & $-47,1^{*}$ & 4,0 \\
\hline Repetición secundaria & & & & & $-13,4$ & 8,0 & $-15,1$ & 7,9 \\
\hline Sexo & & & & & $11,0^{*}$ & 1,6 & $10,7 *$ & 1,6 \\
\hline Nivel educativo & & & & & $32,3^{*}$ & 5,1 & $21,7 *$ & 4,8 \\
\hline Horas de lectura & & & & & $-1,7$ & 1,6 & $-1,5$ & 1,6 \\
\hline Gusto por la lectura & & & & & $8,6^{*}$ & 0,7 & $8,7 *$ & 0,7 \\
\hline $\begin{array}{l}\text { Promedio de ESCS de la } \\
\text { escuela }\end{array}$ & & & & & & & 27,7 * & 2,8 \\
\hline $\begin{array}{l}\text { Desviación estándar de } \\
\text { ESCS de la escuela }\end{array}$ & & & & & & & $-32,1^{*}$ & 12,2 \\
\hline Sostenimiento & & & & & & & $-8,4^{*}$ & 6,9 \\
\hline $\begin{array}{l}\text { Porcentaje de la varianza } \\
\text { en los alumnos }\end{array}$ & & 38,7 & & 41,2 & & 70,2 & & 78,4 \\
\hline $\begin{array}{l}\text { Porcentaje de la varianza } \\
\text { entre escuelas }\end{array}$ & & 61,3 & & 58,8 & & 29,8 & & 21,6 \\
\hline
\end{tabular}

Nota: $*$ p $<0,05$.

Fuente: Elaboración propia con datos de la aplicación de PISA (2009).

De acuerdo a los resultados obtenidos en la varianza explicada entre las escuelas hay un paulatino decremento a lo largo de los diferentes modelos en la escala global de lectura, al igual que en sus subescalas. En los modelos vacíos o nulos el porcentaje de varianza entre escuelas es de aproximadamente el $61 \%$ tanto para la escala global como para las tres diferentes subescalas. En lo que refiere al modelo en el que sólo se incluye como variable ESCS, la varianza entre escuelas disminuye de manera marginal, apenas de dos a tres puntos porcentuales. Cuando se incluyen otras variables como la reprobación, el sexo, el nivel educativo, las horas dedicadas a la lectura y el gusto por la misma el porcentaje de varianza entre escuelas disminuye hasta 30 o menos puntos porcentuales; finalmente, en el modelo donde se incluyen factores contextuales de la escuela, la varianza que se explica llega a ser de aproximadamente $20 \%$; esto es a medida que se incluyen variables explicativas en los modelos las diferencias entre escuelas disminuye gradualmente. 


\section{Discusión}

El principal objetivo del presente trabajo fue analizar el potencial explicativo que tienen algunas variables contextuales de la escuela sobre los resultados obtenidos en la prueba de lectura de PISA 2009, una vez que se controlan algunas de las variables individuales del alumno. Debe señalarse que si bien no se ignoran los efectos de los factores individuales sobre el desempeño de los estudiantes, el estudio estuvo orientado principalmente a la investigación de la incidencia del nivel socioeconómico y cultural de la escuela sobre el logro escolar.

Se examinaron diferentes puntos de interés que sugieren la estructura que siguen los diferentes modelos. El primero de ellos pretendió conocer si las diferencias en el ESCS por sí mismas son suficientes para explicar en gran medida las brechas en el rendimiento, y de no ser así cuál es la contribución que realizan para explicar la variabilidad entre los individuos y las escuelas.

Los resultados obtenidos en los diversos modelos muestran la escasa contribución que el nivel socioeconómico y cultural (ESCS) de la familia hace al desempeño en la escala global de lectura y en cada uno de sus dominios; este resultado resalta si lo comparamos con los efectos que tienen la reprobación de un grado, principalmente en primaria, resultado que es observado en el desempeño escolar en general (Martínez, 2004). En segundo lugar se encuentra el nivel educativo, ya que para esta edad el seguir una trayectoria regular y ubicarse en la educación media superior le significa una ganancia considerable (Robles et al., 2012). De igual forma, los resultados confirman la superioridad de las mujeres por encima de los hombres en las competencias lectoras; un impacto similar al de mostrar un gusto por la lectura. Sobre este último punto debe señalarse el resultado negativo, aunque marginal, que tienen las horas de estudio. Esto puede ser atribuible a que lo que se está reflejando con esta variable es la necesidad de estudio que tienen los estudiantes con baja competencia lectora, a menor competencia mayor necesidad de dedicarse a la lectura. Finalmente, de los efectos contextuales solamente un ambiente favorecedor (el promedio del ESCS de la escuela) es consistente a lo largo de la escala y las subescalas de la prueba de lectura, en tanto que la influencia de la heterogeneidad de la escuela y del tipo de sostenimiento varía en su significatividad.

Claramente, la reprobación en primaria y el efecto contextual del ESCS (el promedio de la escuela) hacen la contribución más importante al logro obtenido en la escala global de lectura y en sus tres diferentes subescalas. Por su parte, tanto el número de horas dedicado a leer como el índice del gozo por la lectura, hacen una contribución modesta, y en ocasiones no significativa, al desempeño en la competencia lectora.

El papel que juegan los centros escolares en la explicación de la variación total del rendimiento en lectura es crucial, donde el peso de las características de las escuelas ya sea como el promedio de los aportes individuales del ESCS de cada alumno o la heterogeneidad de este mismo contexto, es igual o mayor que a muchas de las variables individuales del alumno, con excepción de la reprobación. De tal forma que el logro esperado de cada estudiante depende en gran medida de la escuela, del nivel al que asiste, y, en algunos casos del tipo de sostenimiento de aquélla, en la educación media superior principalmente. La repetición apreciada desde un punto de vista económico significa volver a incurrir en un gasto realizado debido a que el sistema no pudo garantizar el tránsito fluido de un grupo de estudiantes en un año escolar dado (Carrasco, 2008). 
De manera particular, es de señalar que más allá de la dimensión socioeconómica y cultural de la familia, el contexto de la escuela influye de manera decisiva sobre el desempeño escolar. Los resultados presentados en este trabajo no corresponden al hito que el estatus socioeconómico de la familia tiene en el desempeño académico (Coleman et al., 1966), aunque si bien el capital económico permite a los padres proporcionar el acceso a mejores instituciones educativas a sus hijos, usualmente las que tienen una subvención particular o por parte de los padres. En este sentido, Bazán y colaboradores (2007) han señalado que las características de la escuela (referido a los maestros) predicen de forma significativa, en el mismo nivel que el apoyo familiar proporcionado en el aprendizaje, sobre indicadores de logro escolar en competencias de español de alumnos de primaria en escuelas públicas de Sonora, que el nivel educativo y cultural influye en el logro educativo, solamente mediado por el apoyo familiar.

No obstante, la calidad de las escuelas privadas, aunque homogénea en los niveles básicos como consecuencia de que la cobertura en éstos es casi universal por lo que los padres o tutores eligen las escuelas por su calidad, en la educación media superior ante la falta de cobertura educativa por parte del Sistema Educativo Mexicano la cual no le permite atender la demanda de estudiantes de este nivel, hay abundancia de oferta en el ámbito de instituciones privadas que tienen una calidad muy variable en la que los padres optan por las mismas debido a la carencia de opciones (Robles et al., 2013).

El Estado mexicano no puede intervenir sólo a través de políticas educativas para paliar los efectos que el nivel socioeconómico y cultural, ya sea individual y/o contextual, tiene sobre la adquisición y desarrollo de competencias para la vida en las sociedades del conocimiento. La conformación de los grupos escolares en la educación básica tiene un componente de autoselección donde los padres con un capital económico considerable, y una formación cultural y académica usualmente a la par, pueden elegir escuelas de buena calidad académica así como transformar dicho capital económico en uno cultural (Backhoff et al., 2007), brindando a sus hijos oportunidades de aprendizaje que no tienen los alumnos de un extracto económico más bajo y/o que asisten a escuelas públicas. Mediante programas sociales, y extracurriculares, el SEN puede promover la adquisición de las competencias requeridas (lectura, matemáticas y ciencias) a nivel de educación básica.

En contraparte, en el nivel medio superior la prioridad de las políticas del SEN no sólo será la calidad de la educación que se imparta sino la mayor cobertura posible de los alumnos, la cual tiene como objetivo el 100\% para el ciclo escolar 2021/2022 (Robles et al., 2013).

Aunque las variables socioeconómicas y culturales no son controlables directamente a través de decisiones políticas, sí se puede moderar el impacto de éstas en el logro educativo del estudiante para proporcionar una mayor igualdad de oportunidades. El mayor desafío para la política educativa es fomentar altos niveles de logro educativo (calidad), en tanto que limita la influencia del contexto educativo en los resultados del aprendizaje (equidad), lo cual puede ser considerado un indicador de ineficiencia en los sistemas educativos para capitalizar completamente el potencial cognitivo de sus estudiantes (Aydin et al., 2010).

El camino para lograr mejores resultados en la escuela es través de la escuela pública, la cual se ha vuelto imprescindible en las consecuencias de la equidad y la igualdad en el marco del aprendizaje a lo largo de la vida. Esto sugiere una demanda legítima de la 
equidad que debe ser un elemento central de las políticas públicas destinadas a reducir las brechas sociales y económicas. Para acceder a la equidad se logra a través de la diversidad y la inclusión de los sujetos, donde las desigualdades son históricas y estructurales. La inclusión (diversidad) y la participación en la misma (inclusividad), son requeridos en la ciudadanía democrática, y en la implementación de la escuela pública como medio de obtener mejores resultados educativos (Sánchez-Santamaría y BallesterVila, 2014).

\section{Referencias}

Aydin, A., Uysal, Ş. y Sriaer, Y. (2010). Analyzing the results of PISA maths literacy in terms of social justice and equality in educational opportunities. Procedia Social and Behavioral Sciences, 2, 3537-3544. doi: 10.1016/j.sbspro.2010.03.548

Backhoff, E.E., Bouzas, A.R., Contreras, C., Hernández, E.P. y García, M.P. (2007). Factores escolares y aprendizaje en México. El caso de la educación básica. México: INEE.

Backhoff, E.E., Bouzas, A.R., Hernández, E.P. y García, M.P. (2007). Aprendizaje y desigualdad social en México: implicaciones de política educativa en el nivel básico. México: INEE.

Backhoff, E.E., Andrade, E.M., Sánchez, A.M., Peon, M.Z. y Bouzas, A.R. (2006). El aprendizaje del español y las matemáticas en la educación básica en México: sexto de primaria y tercero de secundaria. México: INEE.

Bazán, A., Barrera, D.E. y Vega-Alcantara, N.I. (2013). Validación de constructos de competencias de lectura y producción de textos en los inicios de la generalización de la reforma en la primaria mexicana. REICE. Revista Iberoamericana sobre Calidad, Eficacia y Cambio en Educación, 1 1(4), 61-76.

Bazán, A., Sánchez, B. y Castañeda, S. (2007). Relación estructural entre apoyo familiar, nivel educativo de los padres, características del maestro y desempeño en lengua escrita. Revista Mexicana de Investigación Educativa, 12, 701-729.

Bonamino, A. y Crespo, F. (2004). Eficácia e eqüidade na escola fundamental brasileira. Brasilia: Fondo de Investigaciones Educativas-PREAL.

Carrasco, G.G. (2008). Influencia del capital cultural, capital económico y capital social basado en la familia sobre el rendimiento de los estudiantes: un análisis comparativo. Lima: Consorcio de Investigación Económica y Social. Recuperado de http://cies.org.pe/

Cervini, R. (2002). Participación familiar y logro académico del alumno. Revista Colombiana de Educación, 43, 69-102.

Coleman, J.S., Campbell, E.Q., Hobson, C.J., McPartland,J., Mood, A.M. y Weinfield, F.D.(1966). Equality of educational opportunity. Washington: US Government Printing Office.

Díaz, G.M. y Flores, V.G. (2010). México en PISA 2009. México: INEE.

Hanushek, E.A. (1989). The impact of differential expenditures on school performance. Educational Researcher, 18(4), 45-51. doi: 10.3102/0013189x018004045

Hernández-Castilla, R., Murillo, F.J. y Martínez-Garrido, C. (2014). Factores de ineficacia escolar. REICE. Revista Iberoamericana sobre Calidad, Eficacia y Cambio en Educación, 12(1), 103-118.

Langer, W. (2007). The end of equal opportunities? A multilevel analysis of Luxembourg PISA 2003 Data. Recuperado de http://www.statistiques.public.lu/catalogue-publications/ 
Lee, Y.H. y Wu, J.Y. (2012). The effect of individual differences in the inner and outer states of ICT on engagement in online reading activities and PISA 2009 reading literacy: Employing the relationship between the old and new reading literacy. Learning and Individual Differences, 22, 336-342. doi: 10.1016/j.lindif.2012.01.007

Lizasoain, L., Joaristi, L., Lukas, J.F. y Santiago, K. (2007). El efecto contextual del nivel socioeconómico sobre el rendimiento académico en la educación secundaria obligatoria en la Comunidad Autónoma Vasca (España). Estudio diferencial del nivel socioeconómico familiar y el del centro escolar. Archivos Analíticos de Políticas Educativas, 15(8), 1-37.

López, E.M., Navarro, E.A., Ordoñez, X.C. y Romero, S.M. (2009). Estudio de variables determinantes de eficiencia a través de modelos jerárquicos lineales en la evaluación PISA 2006: el caso de España. Archivos Analíticos Políticos Educativas, 17(6), 1-27.

Martínez, F.R. (2004). ¿Aprobar o reprobar? El sentido de la evaluación en educación básica. Revista Mexicana de Investigación Educativa, 9(23), 817-839.

Organisation for Economic Cooperation and Development-OECD (2010a). PISA 2009 results: what students know and can do student performance in reading, mathematics and science. París: OECD.

Organisation for Economic Cooperation and Development-OECD (2010b). PISA 2009 results: overcoming social background-equity in learning opportunities and outcomes. París: OECD.

Organisation for Economic Cooperation and Development-OECD (2012). PISA 2009 technical report. París: OECD.

Raudenbush, S. y Bryk, A. (2002). Hierarchical linear models: applications and data analysis methods. Pasadena, CA: Sage.

Robles, V., Hernández, J., Peralta, Y., Dander, M., Zendejas, L. y Medrano, V. (2012). Panorama educativo de México 2010. Indicadores del sistema educativo nacional educación básica y media superior. México: INEE.

Robles, V., Hernández, J., Zendejas, L., Pérez, M., Peralta, Y. y Medrano, V. (2013). Panorama educativo de México 2012. Indicadores del sistema educativo nacional educación básica y media superior. México: INEE.

Sánchez, A.M. y Andrade, E.M. (Coords.) (2009). El aprendizaje en tercero de secundaria en México: Informe sobre los resultados del Excale 09, aplicación 2008. Español, Matemáticas, Biología y Formación cívica y ética. México: INEE.

Sánchez, A.M. y Andrade, E.M. (Coords.). (2013). El aprendizaje en sexto de primaria en México. Informe sobre los resultados del Excale 06, aplicación 2009. Español, Matemáticas, Ciencias naturales y Educación cívica. México: INEE.

Sánchez-Santamaría, J. y Ballester Vila, M.G. (2014). Desarrollando el éxito educativo para todos: reflexiones, propuestas y retos conceptuales en torno a la equidad educativa. REICE. Revista Iberoamericana sobre Calidad, Eficacia y Cambio en Educación, 12(2), 85-104.

Sirin, S.R. (2005). Socioeconomic status and academic achievement: a meta-analytic review of research. Review of Educational Research, 75(3), 417-453. doi: 10.3102/00346543075003417

Thorpe, G. (2006). Multilevel analysis of PISA 2000 Reading results for the United Kingdom using pupil scale variables. School Effectiveness and School Improvement, 17(1), 33-62. doi: 10.1080/09243450500264473

Vázquez, R. (2005). La escuela a examen. Las reformas educativas, más de cuatro décadas de fracasos. Observatorio Ciudadano de la Educación, 5(1), 1-7. 
Yip, D.Y. Chiu, M.M. y Ho, E.S. (2004). Hong Kong student achievement in OECD PISA study: gender differences in science content, literacy, skills, and test item formats. International Journal of Science and Mathematics Education, 2, 91-106. doi: 10.1023/b:ijma.0000026537.85199.36 